\title{
Determination of turbulent energy dissipation rate directly from MF-radar determined velocity
}

\author{
C. M. Hall ${ }^{1}$, S. Nozawa ${ }^{2}$, A. H. Manson ${ }^{3}$, and C. E. Meek ${ }^{3}$ \\ ${ }^{1}$ The Auroral Observatory, University of Troms $\phi$, Norway \\ ${ }^{2}$ STE Laboratory, Nagoya University, Japan \\ ${ }^{3}$ Institute of Space and Atmospheric Studies, University of Saskatchewan, Saskatoon, Canada
}

(Received May 17, 1999; Revised October 26, 1999; Accepted December 21, 1999)

\begin{abstract}
MF radar systems are able to determine horizontal neutral winds in the mesosphere and, to some extent in the lower thermosphere by cross-correlations of signals received at spaced antennas. Essentially, by also computing auto-correlations, signal fading may be measured which in turn is thought to be largely attributable to turbulence. Hitherto, estimates of upper limits for the turbulent energy dissipation rate have been derived from the characteristic fading times. In this paper, we propose that power spectra of the velocity components themselves may be used to yield estimates of turbulent energy dissipation rate. 2-minute resolution velocities from the Universities of Saskatchewan, Troms $\varnothing$ and Nagoya joint MF radar at $69^{\circ} \mathrm{N}, 19^{\circ} \mathrm{E}$ are used in a pilot analysis to illustrate and ratify the method.
\end{abstract}

\section{Introduction}

The most common use of MF radar systems is that of determining horizontal winds in the mesosphere (e.g. Reid, 1996; Hocking, 1997). To do this, the atmosphere is illuminated with typically $2-3 \mathrm{MHz}$ and structures in the refractive index generate a diffraction pattern on the ground. If the scattering structures may be assumed to move with the neutral wind, this diffraction pattern moves across the ground with twice that speed. Signals received at spaced antennae are then cross-correlated and the wind speed determined. The analysis method, known as the Full Correlation Analysis, or FCA, is thoroughly described by Briggs (1984). To obtain the results we shall present here, the exact method we use is specified by Meek (1980). Apart from cross-correlating the signals, it is possible to auto-correlate them and obtain characteristic fading times for the signals. That the signals fade is due primarily to the imposition of velocity fluctuations and secondarily to the turbulent dissipation of the structures themselves. Thus, the fading times may be used to obtain a velocity fluctuation that, in turn, is used to make an estimate of the upper limit of the turbulent energy dissipation rate, $\varepsilon$. However, the existence of many scatterers or a turbulent reflecting surface may be expected in a large volume. In such an eventuality, the FCA velocity reflects the average of many scatterers; on the other hand, velocity derivation from only one scatterer with a scale of that of the largest eddies is not consistent with the scenario of Briggs (1984). We envisage that fluctuations in the velocity are due to a number of scatterers of the scale of the largest eddies within the radar volume. This is discussed in great detail by Hocking (1999) and should be considered

Copy right $\odot$ The Society of Geomagnetism and Earth, Planetary and Space Sciences (SGEPSS); The Seismological Society of Japan; The Volcanological Society of Japan; The Geodetic Society of Japan; The Japanese Society for Planetary Sciences. obligatory reading for full appreciation of the assumptions inherent in the results presented here. Despite the assumptions, several workers have used this FCA approach, one of the most recent being Hall et al. (1998b) wherein further references may be found. It is only possible to claim that the upper limit of $\varepsilon$ is determined because it is impossible to exclude that some of the velocity fluctuation is due to buoyancy-scale dynamics. If we were able to assert that the velocity fluctuations corresponded to a characteristic scale size equal to the turbulent outer scale $L_{B}$ then the estimates would be nearer $\varepsilon$ itself. In this study, we shall attempt an approach relying solely on the velocity fluctuations themselves obtained from the crosscorrelation results from the FCA. We shall illustrate our new method by analysing a limited amount of data from the joint Universities of Saskatchewan, Troms $\varnothing$ and Nagoya MF Radar in northern Norway at $69^{\circ} \mathrm{N} 19^{\circ} \mathrm{E}$, hereafter referred to as "PRE" for historical reasons (it was originally referred to as "Partial Reflection Experiment"). This system has been described by Manson and Meek (1991).

\section{Method and Validation}

Here, we shall describe the method fully, illustrating each step with analysis results from the PRE radar. Subsequent to the description by Manson and Meek (1991) better (inverted V) receiver antennae have been installed, and the transmitter unit has been replaced by a solid-state unit with higher power (around $40 \mathrm{~kW} \mathrm{rms).} \mathrm{Earlier,} \mathrm{the}$ integration time prior to the FCA has been 5 minutes but in early 1999 this was experimentally changed to 2 minutes facilitating the approach we use here, as we shall see. For our purposes, we shall work with day-averages, although future implementation could certainly employ both shorter and longer averaging depending on the kind of study. 
First, the FCA analysis is used to obtain 2-minute resolution time series of zonal $(u)$ and meridional $(v)$ components of the neutral wind. In practice, data points may be missing due to poor signal, or interruptions. It would be possible to perform spectral analyses of the irregular time series using the Lomb-Scargle approach (Press and Rybicki, 1989), however we will use an FFT here. The data are interpolated onto a regular 2-minute grid, 2-minute being chosen to match the original nominal sampling and thus avoid implicit suppression of higher frequencies that a finer grid would induce. Thereafter linear least-squares fits are made to the resulting power spectral densities (psd's) in log-log space. The results for 12th April 1999 are shown in Fig. 1. This day has been selected from a limited number of 2-minute resolution days obtained so far and having a high data yield and otherwise at random. The psd's for both zonal and meridional components at 85 and $91 \mathrm{~km}$ are shown. Since the linear fit is performed in log-log space and since the density of points is weighted towards high frequencies, these straight lines represent the gravity wave spectral slopes and may be directly compared with results from other workers, e.g. Manson et al. (1999) wherein further references to previous observations may be found. We have avoided filtering the data prior to obtaining the spectra because this can artificially affect the slopes, as discussed in Hall and Aso (1999). In Fig. 1 we see that the high data point density in the gravity wave regime essentially determines the resulting fitted slope; tidal power has no significant effect on the fit, as can be seen from the $85 \mathrm{~km}$ meridional component. Similarly, there is little evidence of any noise floor in the spectra for the data used here (see Fig. 1); Hall et al. (1998b) have used the noise floor as a means of estimating errors in the "fadingtime" method of estimating turbulent intensity. Although psd's are expected to vary considerably with season and geographic location as outlined by Manson et al. (1999), our values compare very favourably. In Fig. 2, we show height profiles of the day-average spectral slopes for the
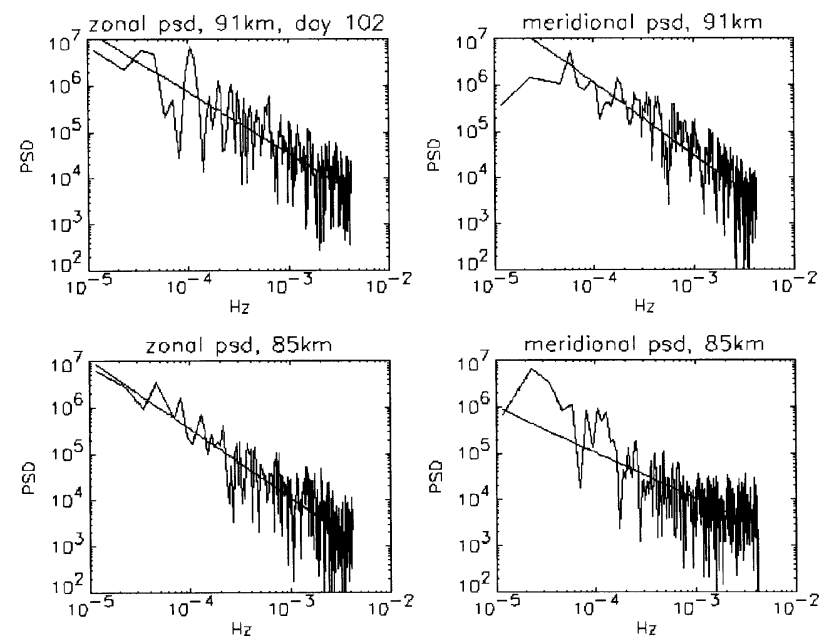

Fig. 1. Power spectral densities of zonal and meridional winds at two heights $(91$ and $85 \mathrm{~km})$ at $69^{\circ} \mathrm{N} 19^{\circ} \mathrm{E}$ on 12 th April 1999. The straight lines represent linear fits to the spectra in log-log space. zonal component. These slopes are a little shallower than the theoretical $-5 / 3$, but this is also consistent with the summer half-year results reported by Manson et al. (1999). Manson et al. (1999) state that, at least for mid-latitude stations, the spectral slopes are near $-5 / 3$ for winter months but closer to -1 for higher frequencies (periods shorter than $2 \mathrm{~h}$ ) in summer and neighbouring equinoctial months. Hall and Aso (1999) found slopes between -1 and -2 in early winter at $78^{\circ} \mathrm{N}$. It is not the objective of this paper, however, to quantify the gravity wave field, so we shall not pursue this aspect further. In Fig. 2, we have shown all heights measured for completeness. Values below $60 \mathrm{~km}$ (poorer signal) and above around $100 \mathrm{~km}$ (danger of significant radio-wave group delay and iondynamics related effects) should be considered less reliable.

We now see the reason for requiring 2-minute resolution data as opposed to the earlier 5-minute resolution: we are now able to identify psd's around the Brunt-Väisälä frequency by having a high density of points both in the presumed buoyancy and inertial dynamic spectral subranges. Although it would have been possible to use 5minute data, extrapolation rather than interpolation would have been incurred whenever the Brunt-Väisälä period was less than $5 \mathrm{~min}$.

Next, we employ the model atmosphere of Hedin (1991) and obtain estimates of the Brunt-Väisälä frequency $\omega_{B}$ as a function of date and altitude. At $\omega_{B}$, we then obtain the corresponding psd on the linear fit and thus the velocity fluctuation by multiplying the psd by $\omega_{B}$ (we consider $\omega_{B}$ to be in rad s${ }^{-1}$, although the frequency axis in Fig. 1 is in $\mathrm{Hz}$ for ease of comparison with other results).

At this point, before utilising the estimated velocity fluctuation, it is appropriate to evaluate the assumptions that have been made. The velocity variance obtained from the FCA-derived velocity at the Brunt-Väisälä frequency

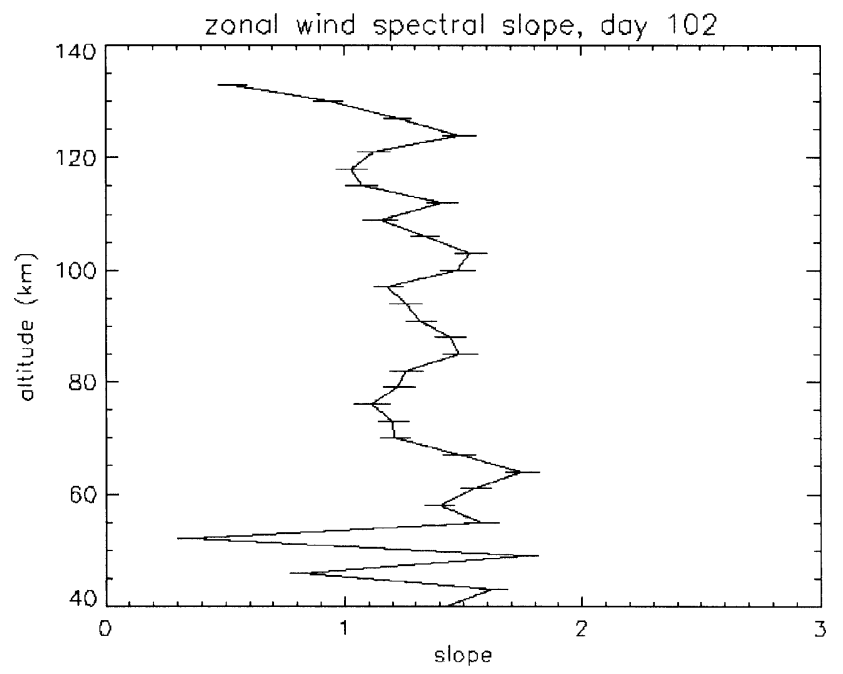

Fig. 2. Day-average spectral slopes for all heights measured and for the zonal wind at $69^{\circ} \mathrm{N} 19^{\circ} \mathrm{E}$ on 12 th April 1999. Low signal levels below $60 \mathrm{~km}$ render the lowest altitude values suspect. Values above around $100 \mathrm{~km}$ may also be questionable due to radio-wave propagation and ionospheric dynamics effects. The error bars are derived from the 1sigma uncertainty in the linear fit. 
represents fluctuations induced by the smallest scale gravity waves. Here, it is assumed that the velocity variance at the Brunt-Väisälä frequency is equal to that at the smallest gravity wave length scales and thus assumed to be equivalent to the velocity variance at the largest eddy scales (assume negligible energy being lost between the smallest wave scale and largest eddy scale). Alternatively, one may think of the turnover time of the largest eddies being equal to the reciprocal of the Brunt-Väisälä frequency (in $\mathrm{Hz}$ ), and in turn equivalent to $\pi L_{B}$ (the eddy circumference) multiplied by the square root of the velocity variance. Herein, any Doppler shifting of the gravity wave frequencies such that they appear to belong to inertial subrange (turbulent) dynamics is ignored. As an aside, it has been suggested that the very Brunt-Väisälä frequency may be identifiable in the spectra as a "bump" (Meek, 1997); as was evident from Fig. 1; this approach would have been inadvisable with our data. Another problem, present in most radar investigation of turbulence, is that the radar volume (i.e. that illuminated by the transmitted beam) is invariably larger, at least in one dimension, than the largest eddies.

Finally, we employ Hocking (1996, equation (27)) to estimate $\varepsilon$ :

$$
\varepsilon=0.47 \sigma^{2} \omega_{B}\left[\frac{1}{c_{f}}\right]^{2 / 3}
$$

where we have used Hocking's (1996) exact notation: $\sigma^{2}$ is the velocity variance at the scale corresponding to $\omega_{B}$. $c_{f}$ is a correction factor to account for beam width and is implicitly unity in the Labitt formalism (Labitt, 1979). The constant 0.47 is explained somewhat by Hocking (1996) but the underlying derivation may be found in Weinstock (1978). Again following Hocking (1996, figure 3 ), we have chosen to use $c_{f}=2.0$ since a typical half-power half beam-width for our system is around 10 $\mathrm{km}$ at $70 \mathrm{~km}$ altitude. Since Eq. (1) is subject to many assumptions (discussed later) and the $c_{f}$ dependence is relatively small, we just assume a constant value for all heights. The minimum dissipation rate supported by the atmosphere, $\varepsilon_{\min }=v \omega_{B}{ }^{2}$ ( $v$ being the kinematic viscosity), corresponds to the energy dissipation due to molecular diffusivity. If there were no turbulence such that the inertial subrange width was infinitesimal, $\varepsilon$ would be identical to $\varepsilon_{\text {min }}$. We subtract $\varepsilon_{\text {min }}$ from our energy dissipation profiles such that we are showing the energy dissipation due to turbulence only (Hall et al., 1998a). Lübken (1996), for example indicates turbulent dissipation by the overshoot of the $\varepsilon$ profile past the $\varepsilon_{\min }$ profile. The resulting estimates of energy dissipation rate (for 12th April 1999) are shown in Fig. 3 (solid lines) using both zonal and meridional winds. The error bars are obtained from the 1-sigma uncertainty in the straight-line fit. The dotted lines (the same on each panel) are the corresponding dissipation rates obtained using the fading time method (e.g. Hall et al., 1998a, b). Comparing Figs. 2 and 3, and the received power (not shown here) we conclude that values below $60 \mathrm{~km}$ are probably unreliable on this particular day.

Another source of error is that in the presence of mean winds, gravity wave frequencies may be Doppler shifted into the spectral regime normally associated with the inertial subrange (i.e. purely turbulent motion) (Gardner et al., 1993). Thus, our estimates of $\varepsilon$ may include buoyant kinetic energy all the same. Since this study is intended as a pilot investigation only, this problem is left as a caveat.

We present data from 10th April 1999 in Fig. 4 and for 6th April 1999 in Fig. 5. On all three days, we see a good agreement between the fading-time (e.g. Hall et al., 1998a) and velocity (this paper) methods. In some ways this is encouraging, but on the other hand the fading time method is supposed to yield upper limits for $\varepsilon$, and not $\varepsilon$ itself. The constants implicit in the two methods are open to question as they generalise as to the state of the atmosphere and
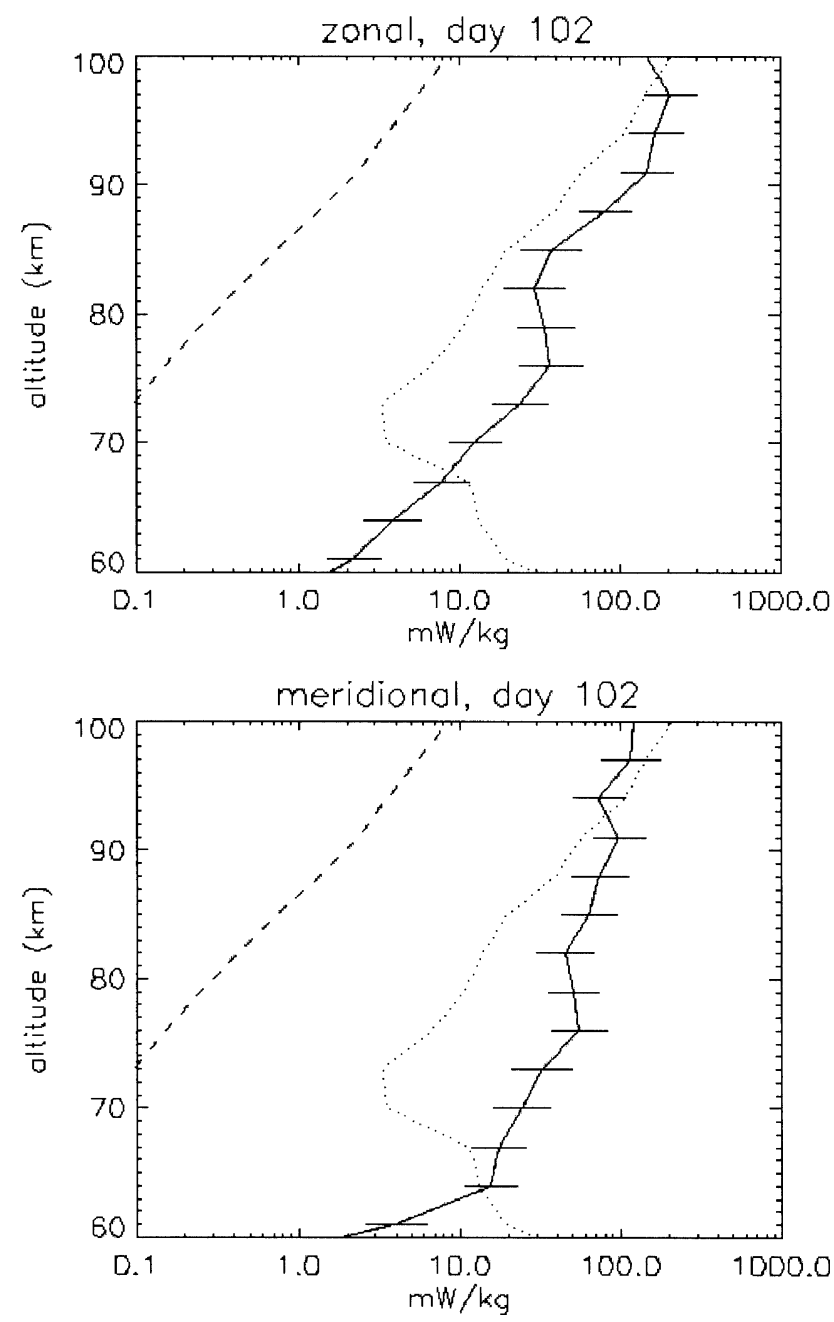

Fig. 3. Derived estimates of turbulent energy dissipation rates for 12 th April 1999 at $69^{\circ} \mathrm{N} 19^{\circ} \mathrm{E}$ (solid lines) for both zonal and meridional winds. The error bars are derived from the 1-sigma uncertainty in the linear fit at the Brunt-Väisälä frequency. The dotted line shows the upper limit estimate for $\varepsilon$ using the classical fading-time method. The dashed line is the energy dissipation rate $\varepsilon_{\min }$ due to molecular diffusion, and therefore the minimum energy dissipation supported by the atmosphere. As opposed to Fig. 2, values below $60 \mathrm{~km}$ and above $100 \mathrm{~km}$ are not shown. 

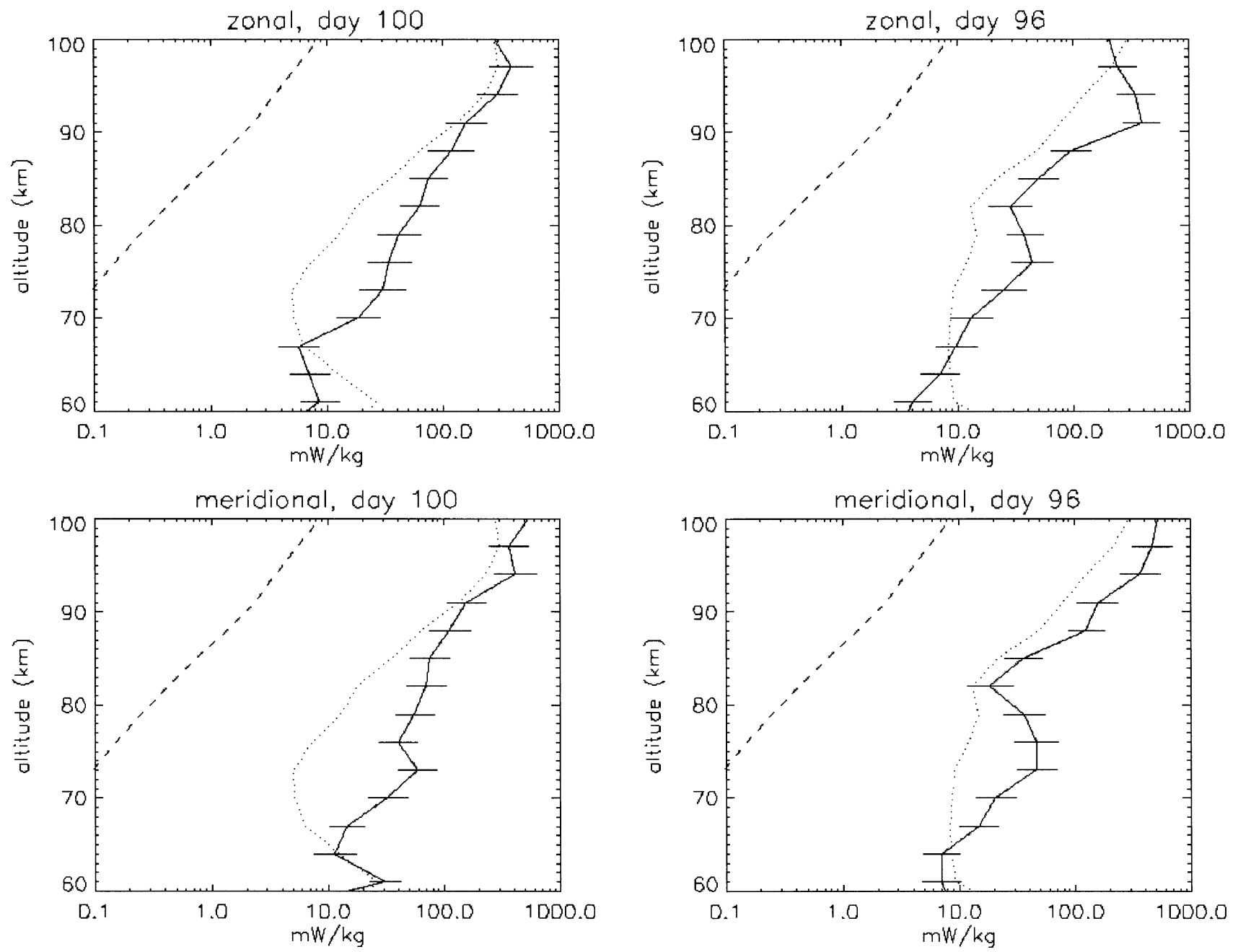

Fig. 4. As for Fig. 3, but for 10th April 1999.

Fig. 5. As for Fig. 3, but for 6th April 1999.

inherently incorporate "typical" flux-Richardson and Prandtl numbers (Weinstock, 1978). An important limitation of using MF radar is that invariably the scattering volume is considerably larger in all dimensions than the buoyancy scale $L_{B}$. The derived velocities (and, for that matter fading times) may arise from detection of only one scatterer, perhaps of the size of the largest turbulent eddy, or from many scatterers filling the volume. Despite the potential errors in the derivation of $\varepsilon$ by the method presented here, the agreement is good compared to other instruments, although high latitude climatological studies of turbulence are sparse. Results from several instruments have been compared recently by Hall et al. (1999), who include the fading-time method profiles included in Figs. 3-5.

\section{Conclusion}

We have used the horizontal velocity measurements from the FCA of MF radar backscatter and estimated velocity fluctuations at the outer or buoyancy scale, $L_{B}$. We propose that these fluctuations are representative of the largest scale turbulent eddies and subsequently apply the theory presented by Weinstock (1978) and described by Hocking (1996) to make estimates of the turbulent energy dissipation rate $\varepsilon$. In contrast to the fading-time method that determines upper limits for $\varepsilon$, these new estimates might be argued to be nearer the true value. However, comparisons show the resulting profiles to be very similar, but then all such measurements enjoy an uncertainty due to spatial intermittency within the scattering volume. Nevertheless, the new method appears robust and perhaps nearer the underlying theory since velocity variance is determined quite directly from the velocity spectra as opposed to the autocorrelations of the signal. In addition, provision of error bars is straightforward; error estimates in fading time are not easily obtained from the FCA. So far, only a limited quantity of 2-minute resolution velocity data (required for this kind of analysis) has been acquired using the joint the Universities of Saskatchewan, Troms $\varnothing$ and Nagoya joint MF radar $\left(69^{\circ} \mathrm{N}, 19^{\circ} \mathrm{E}\right)$. Despite the limited quantity of data used in this pilot investigation, we maintain the method to be as good as the more classical fading-time approach.

Acknowledgments. The authors wish to thank the referees of this paper. The MF radar yields reliable data thanks to the maintenance efforts of Bjørnar Hansen and Bjorn-Ove Husøy. 


\section{References}

Briggs, B. H., The analysis of spaced sensor records by correlation techniques, Handb. MAP, 13, 166-186, 1984.

Gardner, C. S., C. A. Hostetler, and S. Lintelman, Influence of the mean wind field on the separability of atmospheric perturbation spectra, $J$. Geophys. Res., 98, 8859-8872, 1993.

Hall, C. M. and T. Aso, Mesospheric velocities and buoyancy subrange spectral slopes determined over Svalbard by ESR, Geophys. Res. Lett., 26, 1685-1688, 1999.

Hall, C. M., C. E. Meek, and A. H. Manson, Turbulent energy dissipation rates from the University of Troms $\varnothing /$ University of Saskatchewan MF radar, J. Atmos. Solar Terr. Phys., 60, 437-440,1998a.

Hall, C. M., A. H. Manson, and C. E. Meek, Seasonal variation of the turbopause: One year of turbulence investigation at $69^{\circ} \mathrm{N}$ by the joint University of Troms $\varnothing /$ University of Saskatchewan MF radar, $J$. Geophys. Res., 103, 28769-28773, 1998b.

Hall, C. M., U.-P. Hoppe, T. A. Blix, E. V. Thrane, A. H. Manson, and C. E. Meek, Seasonal variation of turbulent energy dissipation rates in the polar mesosphere: a comparison of methods, Earth Planets Space, 51, 515-524, 1999.

Hedin, A. E., Extension of the MSIS thermosphere model into the middle and lower atmosphere, J. Geophys. Res., 96, 1159-1172, 1991.

Hocking, W. K., An assessment of the capabilities and limitations of radars in measurements of upper atmosphere turbulence, $A d v$. Space Res., 17(11), 37-47, 1996.

Hocking, W. K., Strengths and limitations of MST radar measurements of middle-atmosphere winds, Ann. Geophys., 15, 1111-1122, 1997.

Hocking, W. K., The dynamical parameters of turbulence theory as they apply to middle atmosphere studies, Earth Planets Space, 51, 525$541,1999$.
Labitt, M., Some basic relations concerning the radar measurement of atmospheric turbulence, Mass. Inst. of Technol., Lincoln lab., Work. Pap. 46WP-5001, 1979.

Lübken, F.-J., Rocket-borne measurements of small scale structures and turbulence in the upper atmosphere, Adv. Space Res., 17(11), 2535, 1996.

Manson, A. H. and C. E. Meek, Climatologies of mean winds and tides observed by medium frequency radars at Troms $\varnothing\left(70^{\circ} \mathrm{N}\right)$ and Saskatchewan $\left(52^{\circ} \mathrm{N}\right)$ during 1987-1989. Can. J. Phys., 69, 966975, 1991

Manson, A. H., C. E. Meek, C. M. Hall, W. K. Hocking, J. MacDougall, S. Francke, K. Igarashi, D. Riggin, D. C. Fritts, and R. Vincent, Gravity wave spectra, directions and wave interactions: Global MLT-MFR network, Earth Planets Space, 51, 543-562, 1999.

Meek, C. E., An efficient method for analysing ionospheric drifts data, J. Atmos. Terr. Phys., 42, 835-839, 1980.

Meek, C. E., MF radar spaced antenna experiment: wind measurement vs. record length, Atmospheric Dynamics Group Report \#1 1997, University of Saskatchewan, Canada, 32 pp., 1997.

Press, W. H. and G. B. Rybicki, Fast algorithm for spectral analysis of unevenly sampled data, Astrophys. J., 338, 277-280, 1989.

Reid, I. M., On the measurement of gravity waves, tides and mean winds in the low and middle latitude mesosphere and lower thermosphere with MF radar, Adv. Space Res., 18(3), 131-140, 1996.

Weinstock, J., Vertical turbulent diffusion in a stably stratified fluid, $J$. Atmos. Sci., 35, 1022-1027, 1978.

C. M. Hall (e-mail: chris.hall@ @hys.uit.no), S. Nozawa, A. H. Manson, and C. E. Meek 